\title{
A rare collision tumor comprising intracystic papillary neoplasm associated with adenocarcinoma and angiosarcoma in the gallbladder
}

\author{
Geon Park ${ }^{1}$, Won Suk Park ${ }^{2}$, Sang Kuon Lee ${ }^{3}$, and Jong Ok Kim ${ }^{4}$
}

Departments of ${ }^{1}$ Radiology, ${ }^{2}$ Internal Medicine, ${ }^{3}$ Surgery, and ${ }^{4}$ Pathology, Daejeon St. Mary's Hospital, College of Medicine, The Catholic University of Korea, Daejeon, Korea
Received: November 2, 2018

Revised : November 13, 2018

Accepted: November 21, 2018

\author{
Correspondence to \\ Jong Ok Kim, M.D. \\ Tel: +82-42-220-9611 \\ Fax: $+82-42-220-9843$ \\ E-mail:jkim@catholic.ac.kr
}

A 78-year-old woman who had been diagnosed with a gallbladder polyp presented to the emergency department with epigastric pain and dizziness. Nineteen months prior to presentation, a $5 \mathrm{~mm}$-sized enhancing polyp of the gallbladder was first detected on an abdominal computed tomography (CT) $\operatorname{scan}($ Fig. 1A). Seven months prior, the polyp had grown to $2.5 \mathrm{~cm}$ (Fig. 1B). One month prior, the polyp had grown to $3.5 \mathrm{~cm}$, and focal low-density nodular lesions with pericholecystic infiltration were newly detected (Fig. 1C). At the initial diagnosis, the patient had refused a cholecystectomy. On presen-
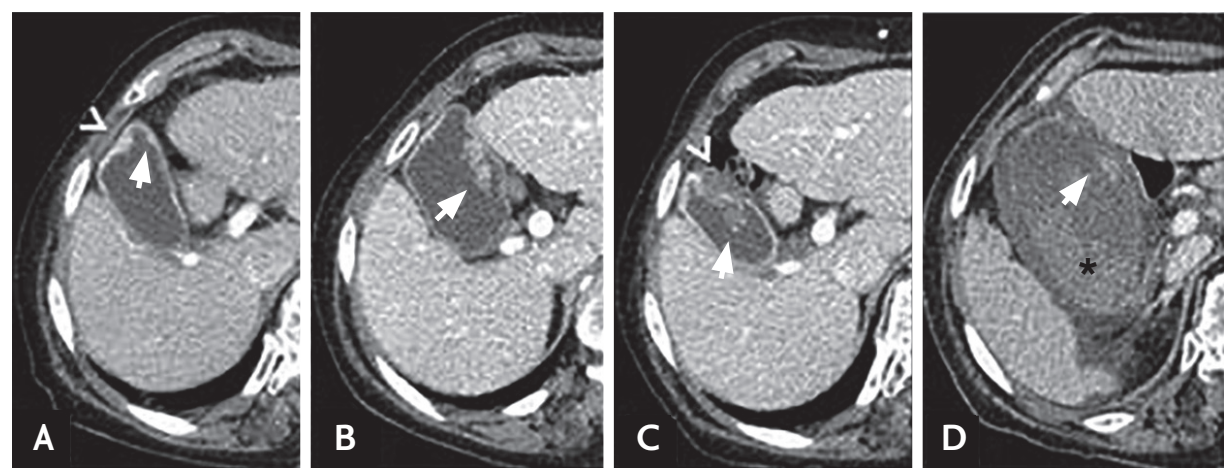

Figure 1. Gallbladder cancer in a 78-year-old woman. (A) Initial enhanced computed tomography (CT) image showing focal wall thickening (arrowhead) and a $5-\mathrm{mm}$ enhancing polyp (arrow) in the fundus of the gallbladder. (B) Enhanced CT image obtained after 12 months showing that the small polyp (arrow) had grown to a $2.5-\mathrm{cm}$ enhancing polypoid mass. (C) Enhanced CT image obtained after 18 months showing that the polypoid mass (arrow) had further enlarged, and focal low density nodular lesions with pericholecystic infiltration were observed (arrowhead). (D) Enhanced CT image obtained after 19 months showing the distended gallbladder with a hyperdense blood clot $\left(^{*}\right)$ in the lumen, diffuse edematous wall thickening, and decreased enhancement of the polypoid mass (arrow). 

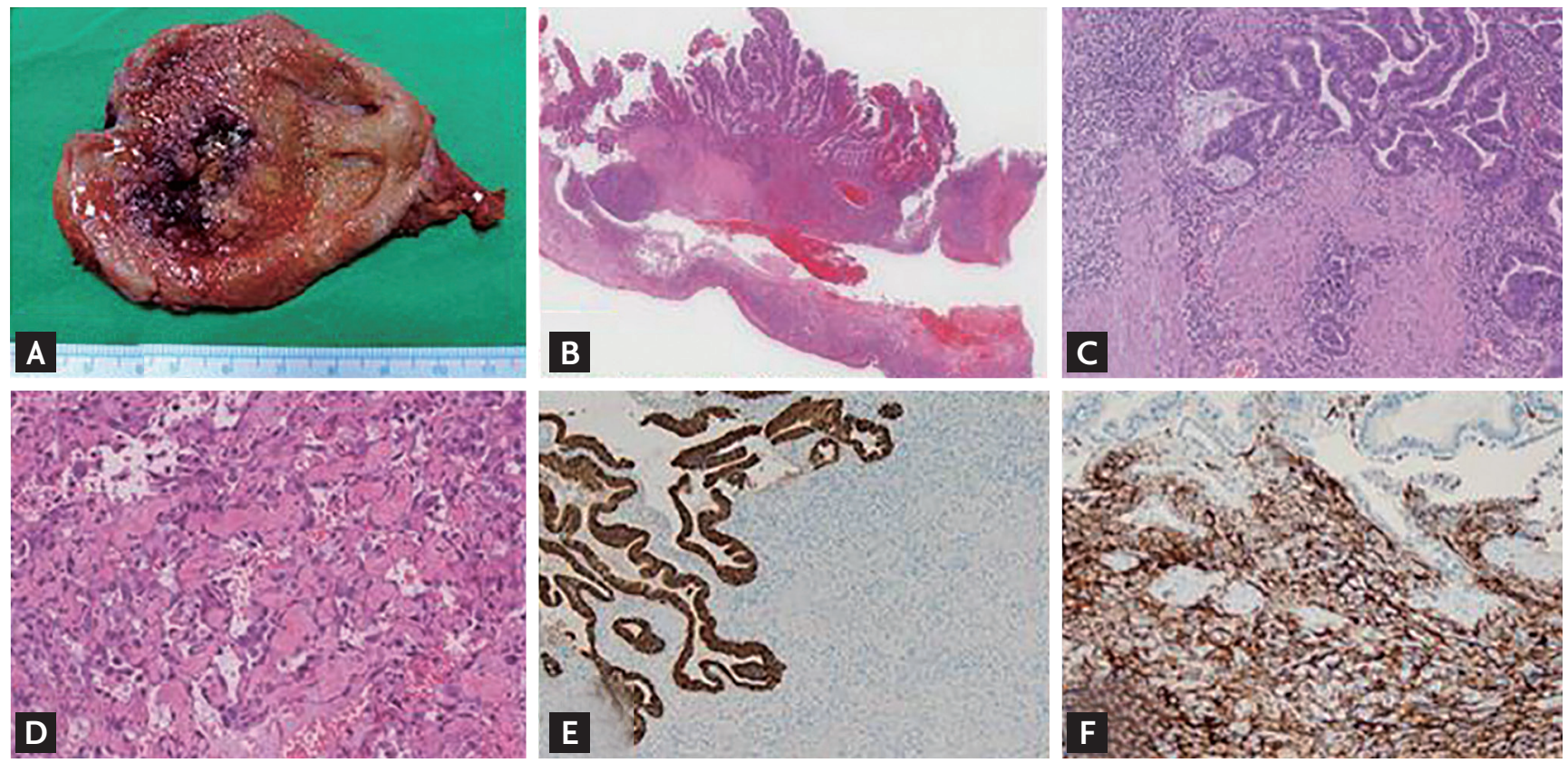

Figure 2. Gross and pathologic findings of intracystic papillary neoplasm associated with adenocarcinoma and angiosarcoma in the gallbladder. (A) A few polypoid masses with hemorrhagic ulcer in the gallbladder. (B) Intracystic papillary neoplasm showing complex papillary configuration, and the solid masses underlying the neoplasm with hemorrhage spreading from the lamina propria to the perimuscular connective tissue $(\mathrm{H} \& \mathrm{E}, \times 7 \mathrm{O})$. (C) Adenocarcinomatous glands invading the muscular layer $(\mathrm{H} \& \mathrm{E}, \times 6 \mathrm{O})$. (D) Angiosarcoma showing anastomosing blood vessels lined by atypical endothelial cells (H\&E, $\times 120)$. (E) Intracystic papillary neoplasm showing immunohistochemical positivity for cytokeratin (×40). (F) Angiosarcoma showing immunohistochemical positivity for $\mathrm{CD}_{31}(\times 200)$.

masses underlying the hemorrhagic mass, measuring 4 $\times 3 \times 2 \mathrm{~cm}$ (Fig. $2 \mathrm{~A})$. The tumor was located $3 \mathrm{~cm}$ away from the resection margin. Pathologically, an intracystic papillary neoplasm (ICPN) with focal adenocarcinoma $(3.5 \times 2.0 \times 0.9 \mathrm{~cm})$ (Fig. $2 \mathrm{~B}$ and $2 \mathrm{C})$ and angiosarcoma $(2.0 \times 2.0 \times 0.3 \mathrm{~cm})($ Fig. $2 \mathrm{D})$ was diagnosed. The ICPN and angiosarcoma were immunopositive for cytokeratin and $\mathrm{CD}_{31}$, respectively (Fig. 2E and 2F). Three months after surgery, the patient is doing well without evidence of recurrence.

Although ICPN is associated with invasive cancer, the prognosis is better than that of other gallbladder can- cers. However, the prognosis of gallbladder angiosarcoma is poor, and the clinical presentation can be mistaken for that of acute cholecystitis. Precise early diagnosis using imaging techniques and pathological examination is important. To our knowledge, this is the only case of collision tumor with adenocarcinoma arising in ICPN and angiosarcoma in the gallbladder.

Written informed consents were obtained.

\section{Conflict of interest}

No potential conflict of interest relevant to this article was reported. 